\title{
ASSESSMENT OF THE RISAT-1 FRS-2 MODE FOR OIL SPILL OBSERVATION
}

\author{
Stine Skrunes, Camilla Brekke, and Martine M. Espeseth \\ UiT The Arctic University of Norway, Department of Physics and Technology, \\ Postbox 6050 Langnes, 9037 Troms $\varnothing$, Norway
}

\begin{abstract}
Synthetic aperture radar data acquired by the Radar Imaging Satellite (RISAT-1) over experimental oil spills is here investigated. One quad-polarization scene in the Fine Resolution Alternate Polarization Stripmap (FRS-2) mode is analyzed to evaluate the potential of using this mode for oil spill observation. Oil slicks of varying type and age are clearly detected in the $\mathrm{HH}$ and VV channels, with relatively high signal-to-noise ratios. The cross-polarization channel is not found useful due to its proximity to the noise floor and some processing issues in the received product. Only intensity-based multipolarization parameters can be extracted due to the incoherent data acquisition. The Total Copolarization Power and the Polarization Difference are found to have good detection capabilities, whereas the Copolarization Power Ratio and the Normalized Polarization Difference only show small indications of the slicks. Comparison between SAR data and coincident observations from aircraft show a correlation between enhanced SAR signatures and locations of thicker oil layers.
\end{abstract}

Index Terms - RISAT-1, oil spill, polarimetry, thickness

\section{INTRODUCTION}

Space borne synthetic aperture radar (SAR) data are used operationally for continuous ocean surveillance and oil spill detection. However, there are limitations on the accuracy of the detection services and on the amount of information that can be extracted. Look-alike phenomena are still a challenge, and there are no well-established methods to retrieve important oil spill properties such as volume and thickness variations from SAR data. Much of the research addressing these questions are looking at the use of multipolarization (multipol.) SAR data, see, e.g., [1] and references therein.

The spatial and temporal resolutions of individual SAR sensors are limited, and hence, the full set of available SAR sensors should be included in the operational oil spill detection services. The Radar Imaging Satellite (RISAT-1), owned by the Indian Space Research Organisation (ISRO) and launched in 2012, is one of the more recently available SAR satellites. RISAT-1 is a C-band SAR sensor and offers

This study is funded by CIRFA partners and the Research Council of Norway (RCN grant no. 237906).
Table 1: Overview of the oil releases present in the RISAT-1 FRS-2 scene. ISB denotes in situ burning, and HISB indicates herder was applied prior to ISB. Disp. denotes dispersion. Åsgård Blend and Grane Blend are both crude oils.

\begin{tabular}{lllcl}
\hline & $\begin{array}{c}\text { Time of } \\
\text { release }\end{array}$ & Oil type & $\begin{array}{c}\text { Volume } \\
{\left[\mathrm{m}^{3}\right]}\end{array}$ & $\begin{array}{l}\text { Clean-up } \\
\text { system }\end{array}$ \\
\hline \hline$\# 1$ & 06.43 & Åsgård Blend & 10 & None \\
$\# 2$ & 08.03 & Åsgård Blend & 10 & Disp. \\
$\# 3$ & 11.21 & Grane Blend & 6 & HISB \\
$\# 4$ & 13.15 & Emulsion & 50 & Booms \\
$\# 5$ & 13.40 & Grane Blend & 6 & ISB, disp. \\
$\# 6$ & 17.07 & Grane Blend & 4 & HISB, disp. \\
\hline
\end{tabular}

a variety of polarization modes, including quad-polarization (QP) and compact polarization (CP) data. Some studies on the use of RISAT-1s CP mode for oil spill observation have been done, see, e.g., [2]. However, to the authors knowledge, the applicability of the QP Fine Resolution Alternate Polarization Stripmap (FRS-2) mode for this purpose has not been investigated before.

The objective of this paper is to evaluate the applicability of RISAT-1 FRS-2 QP data for oil spill detection and characterization. One SAR scene containing experimental oil slicks of various types and ages is investigated, and compared to coincident optical observations from an aircraft.

\section{DATA}

One RISAT-1 FRS-2 scene, acquired during an oil-on-water exercise in the North Sea on the 14 June 2016 is here investigated. The exercise was conducted by the Norwegian Clean Seas Association for Operating Companies (NOFO) and included several releases of oil onto the sea surface to test oil spill clean-up systems such as dispersion and in situ burning.

The investigated FRS-2 scene was acquired in QP (alternating polarization). In this mode, data are collected with a swath width of $25 \mathrm{~km}$, a resolution of $9 \mathrm{~m}$ (azimuth) $\times 4 \mathrm{~m}$ (range), and a noise equivalent sigma zero (NESZ) of -20 dB [3]. The SAR data collection took place at 17.15 UTC, and six oil releases had been done prior to this, see details in Ta- 


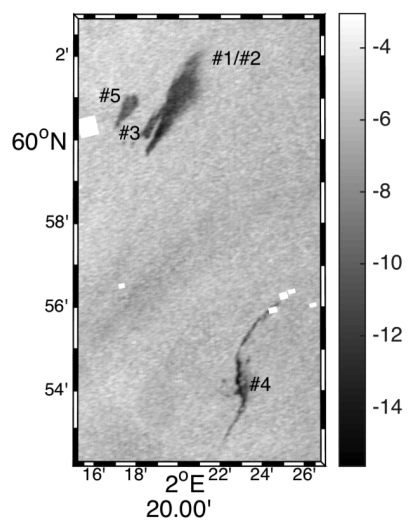

(a) VV intensity.

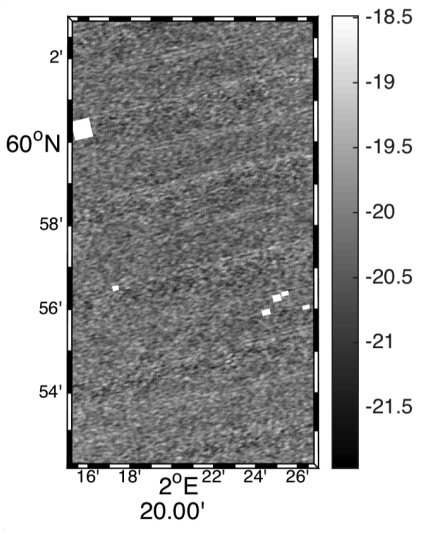

(b) HV intensity.
Fig. 1: Intensity images $[\mathrm{dB}]$. Ships are masked out to enhance the slick-sea contrast (white boxes). RISAT-1@2016Antrix, processed by KSAT, all rights reserved.

ble 1. The last release is not observed in the scene, probably because it was discharged only a few minutes before the SAR acquisition and had not had enough time to spread out sufficiently. Weather observations around the time of the SAR scene indicated wind speeds of 5.7-8.2 $\mathrm{m} / \mathrm{s}$ from a NE direction, and wave heights of about $0.5-1.25 \mathrm{~m}$. Intensity images of the VV and HV polarization channels are shown in Fig. 1, with the slick identities indicated in Fig. 1(a). Note that two of the releases, \#1 and \#2 have merged into one slick. The $\mathrm{HH}$ channel looks very similar to VV and is not included.

An aircraft operated by the Finnish Border Control was flying over slick \#4 about nine minutes after the RISAT-1 overpass, recording a video of the slick, alternating between visual and infrared (IR) wavelengths. IR sensors can provide information about relative thickness, as thick oil appears hot and bright, intermediate thicknesses appears cool and dark, and sheen is not detected. In this study, thickness variations observed in the aircraft data are compared to internal slick zones found in the SAR data.

\section{ANALYSIS AND RESULTS}

As seen in Fig. 1, the remains of the oil releases that are now 3.5 - 10.5 hours old are clearly detected in the copolarization (copol.) channels with good contrasts to the surrounding clean sea. The slicks are not observed in the HV channel, which clearly shows the ships (masked out), and contains little variation in the ocean areas. The NESZ of the FRS-2 mode is given as $-20 \mathrm{~dB}$ [3], which means that the majority of the pixels are very close to, or below, the noise floor in the HV channel. In addition, a stripe pattern is observed across the image in Fig. 1(b). This may be due to a processing error and is currently being looked into [4].

For the copol. channels the backscatter values are well
Table 2: Definitions of the multipol. features investigated.

\begin{tabular}{ll}
\hline Feature & Definition \\
\hline \hline Polarization Difference & $P D=\left\langle\left|S_{V V}\right|^{2}\right\rangle-\left\langle\left|S_{H H}\right|^{2}\right\rangle$ \\
Normalized $P D$ & $N P D=\frac{P D}{\left\langle\left|S_{V V}\right|^{2}\right\rangle+\left\langle\left|S_{H H}\right|^{2}\right\rangle}$ \\
Copol. Power Ratio & $\gamma_{C O}=\frac{\left\langle\left|S_{H H}\right|^{2}\right\rangle}{\left\langle\left|S_{V V}\right|^{2}\right\rangle}$ \\
Total Copol. Power & $T C P=\left\langle\left|S_{V V}\right|^{2}\right\rangle+\left\langle\left|S_{H H}\right|^{2}\right\rangle$ \\
\hline
\end{tabular}

above the NESZ; in the single-look intensities, the slick regions have mean values between $-12 \mathrm{~dB}$ and $-14 \mathrm{~dB}$ in both the $\mathrm{HH}$ and $\mathrm{VV}$ channel. This is an important advantage if we want to evaluate slick characteristics in addition to detecting the slicks. The possible processing error may be present also in the copol. channels, but is not observed visually in these images.

\subsection{Multipol. parameters and comparison to IR}

Multipol. SAR data have been extensively applied over the last decade for oil spill characterization research, see, e.g., [1] and references therein. The FRS-2 QP mode of RISAT-1 is incoherent, and hence, many traditional multipol. parameters, requiring both intensity and relative phase information, can not be retrieved. Hence, only intensity-based parameters are here extracted from the two copol. channels. The HV channel is excluded due to the proximity to the NESZ and the possible processing error. The investigated parameters are defined in Table 2, where $\left|S_{H H}\right|^{2}$ and $\left|S_{V V}\right|^{2}$ denote the intensity of the $\mathrm{HH}$ and VV channel, respectively, and $\langle\cdot\rangle$ indicates spatial averaging.

In the scattering model described in [5], the backscatter is expressed as the sum of one polarization-dependent component associated with the two-scale Bragg scatter model, and one nonpolarized component. The latter is removed when calculating the Polarization Difference $(P D)$. The $P D$ is therefore controlled by the surface wave components close to the Bragg wavenumber and should reveal near-surface wind variability and presence of slicks [5]. The $P D$ has been evaluated for oil spill observation in, e.g., $[5,6]$ and found to give very good slick-sea contrasts.

Within the tilted Bragg model, the Copol. Power Ratio $\left(\gamma_{C O}\right)$ is independent of the ocean wave spectrum and will only be a function of the dielectric properties, the incidence angle and the slope due to longer ocean waves, which is largely unaffected by oil. A difference in $\gamma_{C O}$ between clean sea and oil slicks indicates that the reduction in backscatter is at least partly due to differences in the dielectric constant [7]. The same would be valid for the normalized $P D, N P D$. $\gamma_{C O}$ has been investigated for oil spill observation in, e.g., $[1,5,6,7]$. In [7], the $\gamma_{C O}$ was used to estimate the oil concentration in oil-water mixtures. The last multipol. parameter 


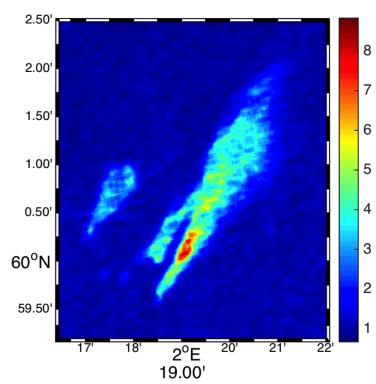

(a) $\zeta_{V V}$.

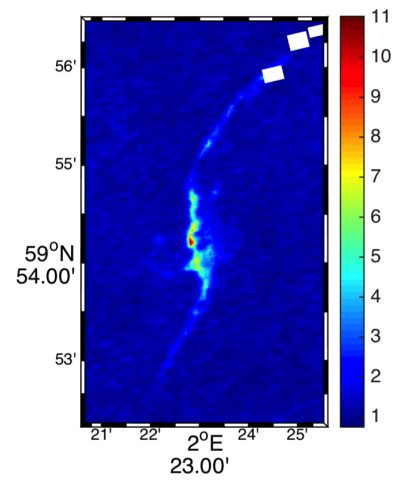

(e) $\zeta_{V V}$.

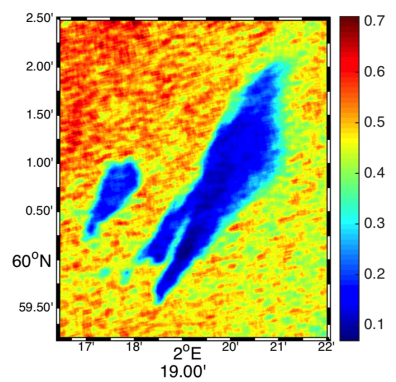

(b) $T C P$.

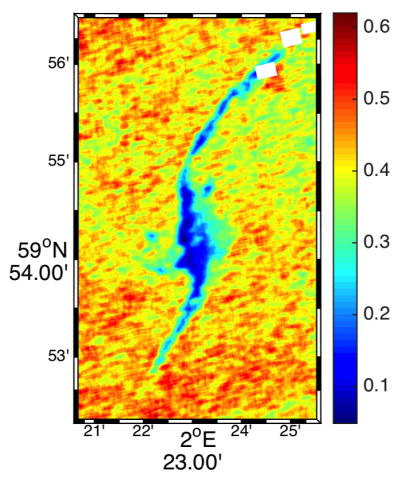

(f) $T C P$.

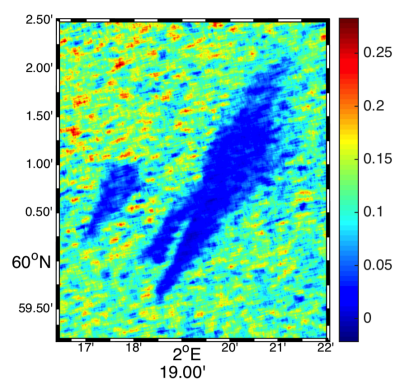

(c) $P D$.

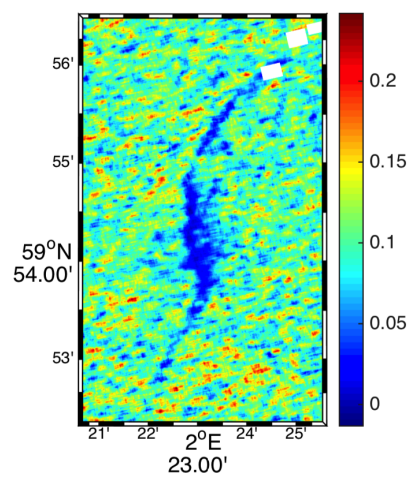

(g) $P D$.

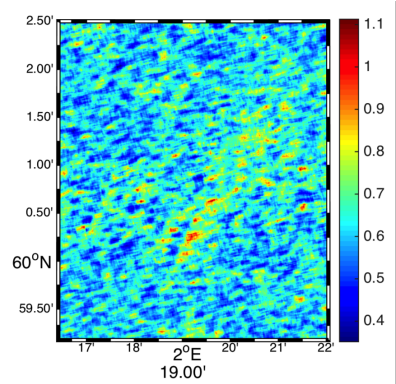

(d) $\gamma_{C O}$.

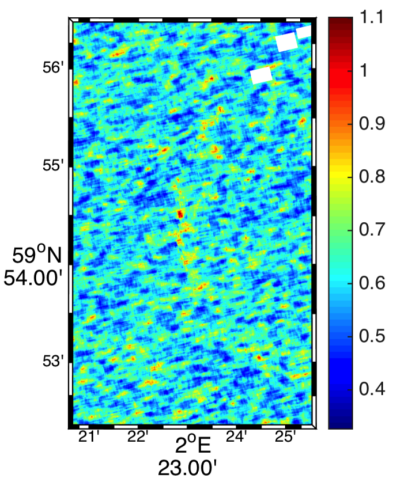

(h) $\gamma_{C O}$.

Fig. 2: Features calculated for two subimages covering the oil slicks. Ships are masked out to enhance the slick-sea contrast.

evaluated in this study is the Total Copol. Power $(T C P)$, which is similar to the span, but is based only on copol. channels. The TCP, $P D$ and $\gamma_{C O}$ are plotted in Fig. 2, in addition to the damping ratio, $\zeta_{V V}$, in which each pixel in $\left\langle\left|S_{V V}\right|^{2}\right\rangle$ is divided by the mean $\mathrm{VV}$ intensity in a clean sea region. $N P D$ looks similar to $\gamma_{C O}$ and is not included.

The $\zeta_{V V}$ shows clear variations within the slicks, including areas with especially strong damping, e.g., in the lower part of the \#1/\#2 slick (upper row) and in the middle part of slick \#4 (bottom row). The damping decreases towards the edges. The TCP seems to detect the full slick regions with high contrasts to the sea, with similar, but less pronounced, spatial variations as $\zeta_{V V}$. $P D$ also provides a good detection, but differs somewhat from the TCP as it seems to exclude the outermost zone observed in TCP. The $\gamma_{C O}$ (and $N P D$ ), look very different from the other parameters, with only small indications of the presence of oil. These may be areas where the dielectric constant is affected by the presence of oil, due to thicker oil layers or higher concentration of oil in the water column. For the rest of the slick, the oil layer may not be thick enough to affect the dielectric constant of the surface, and in turn the $\gamma_{C O}$. The areas of increased $\gamma_{C O}$ are found in locations close to the stronger contrast in $T C P$ and $\zeta_{V V}$.

From the aircraft data, information about the position of thick oil could be retrieved and compared to the internal slick variations observed in the SAR data. We here limit the discussion to slick \#4, which was overflown by the aircraft about nine minutes after the SAR overpass. Fig. 3 shows the $\zeta_{V V}$ from Fig. 2(e) overlaid with the aircraft flight track as small dots. Aircraft positions where thick oil (IR white) was observed in front of the aircraft are plotted as white dots, whereas positions where only thin oil or sheen was observed are indicated by pink dots. The positions of the thick oil are estimated from the position of the aircraft and camera angles, and plotted as white $\times$ 'es. Some uncertainty applies as the information are not logged continuously, and the thick slick is not always located exactly in the middle of the aircraft image. However, the parts of the oil slick where thick oil is observed in IR clearly overlap with the section producing the highest SAR signal damping. Similar relationship between signal damping and thickness has been suggested in some previous studies, e.g., [8]. The thick oil regions also overlap with the area of higher contrast in TCP, PD and $\gamma_{C O}$.

A similar comparison to aircraft data for the most pronounced area in slick \#1/\#2 in Fig. 2(a) has not been carried out yet, as a longer temporal difference complicates the analysis due to drift and weathering. However, aerial data shows that about 1 hour before the SAR acquisition, thick oil was found in the southernmost and western part of the slick, which corresponds well with the observations in Fig. 2(a). This part of the data set should be further investigated in the future. 


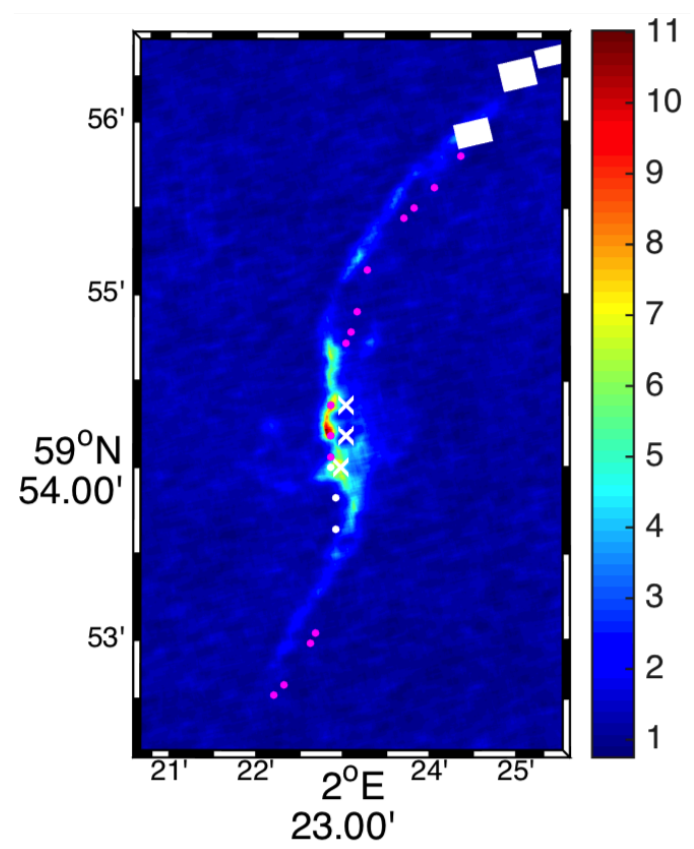

Fig. 3: $\zeta_{V V}$ from the RISAT-1 scene overlaid with the aircraft flight track. White (pink) dots indicate aircraft positions where thick oil slick sections were observed (not observed) in front of the aircraft. White $\times$ 'es indicate positions of the thick oil sections estimated from the aircraft data.

\section{CONCLUSIONS}

The capability of RISAT-1 FRS-2 data for oil spill detection and characterization is here demonstrated for one scene acquired over experimental oil releases. Oil slicks of varying type and age are well detected in the $\mathrm{HH}$ and VV channels, with relatively high signal-to-noise ratios. The crosspol. channel lies close to the noise floor and is dominated by some processing issues, which pose an unknown uncertainty on the operational use of the RISAT-1 FRS-2 data.

Although the data are provided in QP, only intensitybased multipol. parameters can be extracted due to the incoherent alternating polarization acquisition. Hence, multipol. parameters based only on $\left\langle\left|S_{V V}\right|^{2}\right\rangle$ and $\left\langle\left|S_{H H}\right|^{2}\right\rangle$ are here investigated. Both single-polarization damping ratio, and dual-copol. parameters $T C P$ and $P D$ give a good slick detectability, and interesting internal zoning. The areas of highest contrast, particularly in $\zeta_{V V}$, clearly overlaps with areas containing thick oil as observed by aircraft IR, suggesting this type of SAR data may be useful for detecting slick thickness variations. The $\gamma_{C O}$ and NPD only show small indications of the slicks, which may be the areas thick enough to affect the dielectric properties of the surface.

\section{ACKNOWLEDGEMENT}

We thank NOFO for letting us participate in the exercise, and NOFO, SINTEF, and the Norwegian Coastal Administration for providing in situ information. RISAT-1(C)2016-Antrix, processed by KSAT, all rights reserved. Aircraft data are provided by the Air Patrol Squadron Finland/SYKE.

\section{REFERENCES}

[1] Skrunes, S., Brekke, C., and Eltoft, T., "Characterization of marine surface slicks by Radarsat-2 multipolarization features," IEEE Trans. Geosci. Remote Sens., vol. 52, no. 9, pp. 5302-5319, Sep. 2014.

[2] Jayasri, P. V., Sundari, H. S. V. U, Kumari, E. V. S. S., and Prasad, A. V. V., "Study of oil spill in Norwegian area using decomposition techniques on RISAT-1 hybrid polarimetric data," The International Archives of the Photogrammetry, Remote Sensing and Spatial Information Sciences, Vol. XL-8, 2014.

[3] Misra, T., Rana, S. S., Desai, N. M., Dave, D. B., Rajeevjyoti, Arora, R. K., Rao, C. V. N., Bakori, B. V., Neelakantan, R., and Vachchani, J. G., "Synthetic Aperture Radar payload on-board RISAT-1: configuration, technology and performance", Current Science, vol. 104, no. 4, p. 446-461, Feb. 2013.

[4] National Remote Sensing Centre, Indian Space Research Organisation, Personal communication, 2016.

[5] Kudryavtsev, B. Chapron, A. Myasoedov, F. Collard, and J. Johannessen, "On dual co-polarized SAR measurements of the ocean surface," IEEE Geosci. Remote Sens. Lett., vol. 10, no. 4, pp. 761-765, Jul. 2013.

[6] Skrunes, S., Brekke, C., Eltoft, T., and Kudryavtsev, V., "Comparing near coincident C- and X-band SAR acquisitions of marine oil spills," IEEE Trans. Geosci. Remote Sens., vol. 53, no. 4, pp. 1958-1975, Apr. 2015.

[7] Minchew, B., Jones, C. E., and Holt, B., "Polarimetric analysis of backscatter from the Deepwater horizon oil spill using L-band synthetic aperture radar," IEEE Trans. Geosci. Remote Sens., vol. 50, no. 10, pp. 38123830, Oct. 2012.

[8] Wismann, V., Gade, M., Alpers, W., and Hühnerfuss, H., "Radar signatures of marine mineral oil spills measured by an airborne multi-frequency radar," Int. J. Remote Sens., vol. 19, no. 18, pp. 3607-3623, 1998. 